\title{
Comparison of Different Approaches to Include Connection Elements into Frequency-Based Substructuring
}

\author{
Ahmed El Mahmoudi ${ }^{1}$ (D) . Daniel J. Rixen ${ }^{1} \cdot$ Christian H. Meyer $^{1}$
}

Received: 3 July 2019 / Accepted: 10 January 2020 / Published online: 26 March 2020

(C) The Author(s) 2020

\begin{abstract}
Dynamic substructuring (DS) is a research field that has gained a great deal of attention in both science and industry. The aim of DS techniques is to provide engineers in structural vibrations and sound practical solutions for analyzing the dynamic behavior of complex systems. This paper addresses the singularity problem that occurs when flexible joints are implemented as substructures into the Lagrange Multiplier Frequency-Based Substructuring (LM-FBS) coupling process. For illustration, we use rubber bushings from an automotive application. Considering the rubber isolators to exhibit hysteretic damping, we assume that only the property of the dynamic stiffness of material is given. To avoid singularity appearing in the admittance when inverting the impedance of a massless joint, we compare three different approaches to include rubber bushings in the framework of LM-FBS. One method consists in including the dynamic stiffness of material directly in the space of the interface constraints and add it to the assembled interface flexibility of the LM-FBS equation. This corresponds to a relaxation of the interface compatibility condition. In the second method, the rubber bushing is treated as a substructure by adding small masses to the equation of the joint. As a result, we obtain a nonsingular total dynamic stiffness matrix that can be included in the coupling process. The third method describes a novel extension of the LM-FBS approach, based on a solution for singular problems. If the applied forces are self-equilibrated with respect to the rigid body modes, a solution for the singular dynamic stiffness matrix exists. The methods are outlined, both mathematically and conceptually, based on a notation commonly used in LM-FBS. They facilitate the integration of connecting elements together with experimental or numerical determined system dynamics of substructures in order to predict the assembled system behavior.
\end{abstract}

Keywords Dynamic substructuring · Frequency-based substructuring · Connection elements · Compliant interface · Isolators

\section{Introduction}

In complex mechanical structures, it is often difficult to analyze the structural behavior of individual elements independently of each other. Dynamic substructuring (DS) methods enable to deal with this complexity by dividing

\section{Ahmed El Mahmoudi \\ a.mahmoudi@tum.de \\ Daniel J. Rixen \\ rixen@tum.de}

Christian H. Meyer

christian.meyer@tum.de

1 Chair of Applied Mechanics, Faculty of Mechanical Engineering, Technical University of Munich, Boltzmannstr. 15, Garching, 85748, Germany a system into several subsystems, which makes a separate investigation possible. Such methods have been developed and continuously expanded for decades $[1,2]$. In the different DS techniques, the system behavior is derived from the equations of motion of the system. Here, a distinction is made between the methods in the time domain, modal domain and frequency domain. For the time domain, the Direct Coupling method uses the system mass, damping and stiffness to couple the subsystems together. This approach can only be applied if a numerical model of the components are known and are not too big to be handled efficiently (Fig. 1).

In the modal domain, the component mode synthesis (CMS) method is used to couple the different subsystems on the basis of their modal parameters [1]. The CMS method gained particular popularity in the engineering community as a reduction method for finite element models [3]. The basic idea is that the dynamic behavior of the total system 
Fig. 1 Schematic representation of vehicle subcomponents
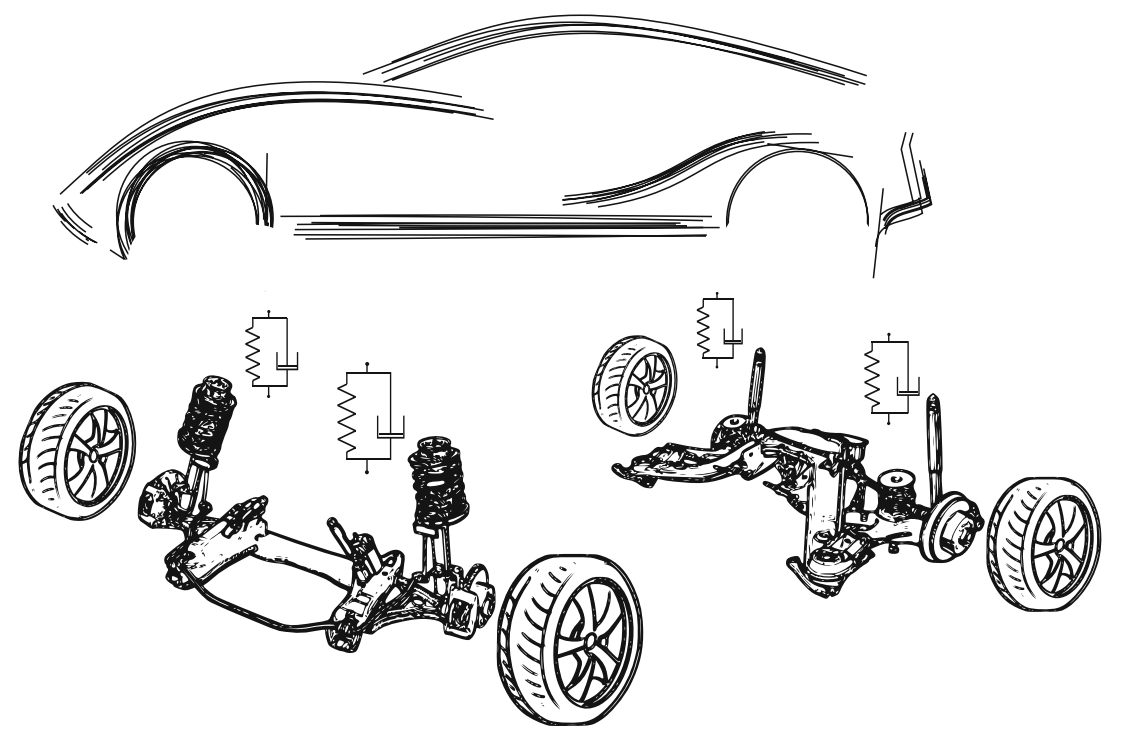

can be described by a limited number of eigenmodes with low damping. In particular, it is used in the context of experimental modal analysis to describe the dynamic behavior of vehicle structures [4].

For the frequency domain, different frequency-based substructuring (FBS) methods are provided, which are not discussed in detail in this article. A detailed overview is provided by [3]. The next sections address the so-called Lagrange multiplier-frequency-based substructuring (LMFBS) method. In this method, the coupling between the subsystems is based on calculated or measured frequencydependent transfer functions, also known as frequency response function (FRF). It is basically suitable for describing the behavior of substructures with high mode density and damped linear systems [3].

Subject of this work is the investigation of different methods to integrate connection elements into the LM-FBS coupling process. The presented methods enable the consideration of experimentally and numerically determined system dynamics of substructures in order to predict the assembled system behavior. Here, rubber bushings are considered as connecting elements. They are frequently used in engineering with the main goal being to reduce the vibration transmission. For instance, they are used between the powertrain and the chassis in cars or in naval construction as isolation support for power units. When considering the coupling elements, it is assumed that the main contribution of the rubber bushings to the assembly is determined by its static stiffness and its damping. Therefore, their inertia is neglected and often not known. Applying the classical LM-FBS method, the absence of inertia causes a singularity problem.

For a better understanding, this paper first gives a brief explanation of the FBS theory in Section "Frequency-Based Substructuring". Subsequently, the different methods of including connection elements into the LM-FBS process are presented in Section "Introduction of Bushings in the General Framework oF LM-FBS". Section "Application of the Methods on Simulated Vehicle Subsystems" continues with an application of the different methods on simulated vehicle subsystems. Finally, a conclusion is given in Section "Conclusions".

\section{Frequency-Based Substructuring}

In this part, the principles of frequency-based substructuring (FBS) will be introduced. The procedure and the use of frequency response functions (FRF) are explained on the basis of the classical theory. The classical theory according to Jetmundsen [5] provides a general formulation to calculate coupled subsystems. In application, however, the equation is intricate, since both mechanical interpretation and implementation are not intuitive [6]. An extension of the formulation to the Lagrange multiplier-frequencybased substructuring (LM-FBS) method yields a more straightforward formulation and thus makes it suitable for application in simulation and experiments. The transmission properties between input and output variables of the individual subsystems are important ingredients for this method. In contrast to classical theory, the LM-FBS method is based on the admittance matrix

$\boldsymbol{Y}^{(s)}=\boldsymbol{Z}^{(s)^{-1}}=\left(\boldsymbol{K}^{(s)}+\boldsymbol{C}(\boldsymbol{i} \boldsymbol{\omega})^{(s)}-\omega^{2} \boldsymbol{M}^{(s)}\right)^{-1}$

of the subsystems. The dynamic stiffness matrix $\boldsymbol{Z}^{(s)}$ is composed of its mass matrix $\boldsymbol{M}^{(s)}$, damping matrix $\boldsymbol{C}^{(s)}$ and stiffness matrix $\boldsymbol{K}^{(s)}$. The dynamic equation for the uncoupled system can be written in impedance form

$Z u=f+g$ 
or in admittance form

$\boldsymbol{u}=\boldsymbol{Y}(\boldsymbol{f}+\boldsymbol{g})$,

where

$\boldsymbol{Z}=\left[\begin{array}{cccc}\boldsymbol{Z}^{(1)} & \mathbf{0} & \cdots & \mathbf{0} \\ \mathbf{0} & \boldsymbol{Z}^{(2)} & & \mathbf{0} \\ \vdots & & \ddots & \\ \mathbf{0} & \mathbf{0} & & \boldsymbol{Z}^{(N)}\end{array}\right], \quad \boldsymbol{u}=\left[\begin{array}{c}\boldsymbol{u}^{(1)} \\ \vdots \\ \boldsymbol{u}^{(N)}\end{array}\right]$

$\boldsymbol{f}=\left[\begin{array}{c}\boldsymbol{f}^{(1)} \\ \vdots \\ \boldsymbol{f}^{(N)}\end{array}\right] \quad$ and $\quad \boldsymbol{g}=\left[\begin{array}{c}\boldsymbol{g}^{(1)} \\ \vdots \\ \boldsymbol{g}^{(N)}\end{array}\right]$.

$\boldsymbol{Z}$ is the block-diagonal dynamic stiffness matrix consisting of the dynamic stiffness matrices of the individual subsystems, $\boldsymbol{u}$ is the vector containing the sets of substructure degrees of freedom (DOF), $\boldsymbol{f}$ is the vector of externally applied forces and $\boldsymbol{g}$ is the vector of interconnecting forces between substructures. All variables are dependent on frequency, that is, for each frequency there is a specific and unique dynamic stiffness matrix. In order to couple the $N$ substructures, two conditions must be satisfied:

- The compatibility condition governs the compatibility of the substructures' displacements at the interface, and can be expressed by

$B \boldsymbol{u}=\mathbf{0}$.

The matrix $\boldsymbol{B}$ operates on the interface DOFs and is a signed Boolean matrix if the interface DoF match. The compatibility condition states that any pair of matching interface DoF $u^{(k)}$ and $u^{(l)}$ must have the same displacement, i.e., $u^{(k)}-u^{(l)}=0$.

- The equilibrium condition on the interface

$g=-B^{T} \lambda$

states that the interface forces are self-equilibrated (action equal reaction). Here $\lambda$ is a set of Lagrange multipliers, denoting the intensity of the interface forces.

By inserting Eq. 6 in Eqs. 2 and 3, one obtains the following impedance and admittance form of the dynamic behavior

$\boldsymbol{Z} \boldsymbol{u}=\boldsymbol{f}-\boldsymbol{B}^{T} \boldsymbol{\lambda}$,

$\boldsymbol{u}=\boldsymbol{Y}\left(\boldsymbol{f}-\boldsymbol{B}^{T} \lambda\right)$.

The dynamic equations for the uncoupled system in the impedance form Eq. 7 and the compatibility condition (5) can be written in block matrix form

$\left[\begin{array}{cc}\boldsymbol{Z} & \boldsymbol{B}^{T} \\ \boldsymbol{B} & 0\end{array}\right]\left[\begin{array}{l}\boldsymbol{u} \\ \boldsymbol{\lambda}\end{array}\right]=\left[\begin{array}{l}\boldsymbol{f} \\ \mathbf{0}\end{array}\right]$.

Inserting the dynamic solution (8) in the compatibility constraint (5), one finds the dual interface problem of the assembly determining the interface forces. The dual interface problem can now be used to compute the interface Lagrange multipliers $\lambda$ :

$\lambda=\left(\boldsymbol{B} \boldsymbol{Y} \boldsymbol{B}^{T}\right)^{-1} \boldsymbol{B} \boldsymbol{Y} \boldsymbol{f}$

Inserting the Lagrange multiplier vector $\lambda$ into the admittance form of the dynamic (8), one obtains the assembled system response $\boldsymbol{u}_{\text {assembled }}$.

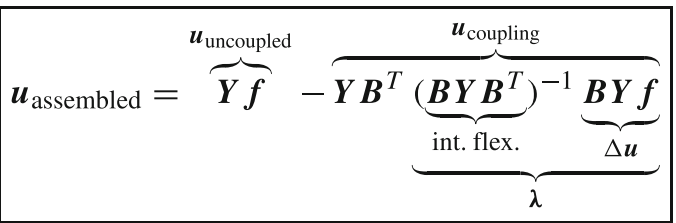

This final result can be interpreted as follows: if an external force $\boldsymbol{f}$ acts on the uncoupled system, a response $\boldsymbol{u}_{\text {uncoupled }}$ is produced. The response $\boldsymbol{u}_{\text {uncoupled }}$ creates an interface incompatibility gap $\Delta \boldsymbol{u}$. In order to close this incompatibility gap on the interface, an interface force $\boldsymbol{g}$ of amplitude $\lambda$ must exist. The term for interface flexibility appears like spring elements in series. Because of the force on the interface $\boldsymbol{g}=-\boldsymbol{B}^{T} \boldsymbol{\lambda}$, a response in the substructure $\boldsymbol{u}_{\text {coupling }}$ is produced. Physically, the dually assembled response $\boldsymbol{u}_{\text {assembled }}$ in Eq. 11 can be interpreted as the combined response to external forces exciting the uncoupled system and to the internal interface forces needed to keep the substructures together while being excited by the external forces.

\section{Introduction of Bushings in the General Framework of LM-FBS}

In this section, the theory of the different methods for integrating of rubber bushings within the LM-FBS approach is explained. Section "Bushings as Compatibility Relaxation" describes a method in which bushings are not implemented as a substructure, but rather are considered as compatibility relaxation to the LM-FBS approach. Subsequently, two methods are presented in which bushings are implemented as substructures within the LM-FBS approach. This is realized in Section "Bushings as Substructure by Adding Virtual Masses" by introducing virtual masses. In Section "Bushing as Substructure via Pseudoinverse" the bushings are implemented as substructures via a pseudoinverse method. Finally, in Section "Comparison of All Three Methods on a Minimal Example" all three methods are compared using a minimal example.

\section{Bushings as Compatibility Relaxation}

The first approach shows a method in which the rubber bushings are considered as connecting elements between 
two substructures, as schematically depicted in Fig. 2. The subscript $i$ and $b$ denote the internal DOF of the substructures and the boundary DOF respectively. Already at early times of the FBS, compliance in the interfaces was considered in the context of the FBS procedure by Jetmundsen [5]. Furthermore, the LM-FBS also dealt with this in [7, 8]. Instead of assuming that the substructures are rigidly connected, as in the classical LM-FBS approach considered by the compatibility condition (5), now the interface compatibility is relaxed by the flexibility of the bushing so that the condition can be written

$B \boldsymbol{u}=\boldsymbol{\delta} \neq \mathbf{0}$.

This allows an interface gap $\delta$ due to the rubber bushings and introduces in the bushing internal forces $\lambda$ proportional to the dynamic stiffness of the bushing. Taking into account the dynamic flexibility matrix $\boldsymbol{\Gamma}$ of the bushing between its interface DOF, Eq. 12 can be completed by

$B \boldsymbol{u}=\delta=\Gamma \lambda$.

It should be noted that $\Gamma$ is not equal to the inverse of the dynamic stiffness matrix of the bushing $\boldsymbol{Z}^{(\text {Bush })^{-1}}$ since it expresses the flexibility related to interface gaps (hence in the space of the internal forces) and not directly the flexibility between the interface DOFs. Considering the model depicted in Fig. 2, the dynamic flexibility matrix $\boldsymbol{\Gamma}$ of the bushing for the two dimensional case, assuming the two directions of the bushing to have equal stiffness and to be uncoupled, can be written

$\boldsymbol{\Gamma}=\left[\begin{array}{cc}\frac{1}{k^{(\text {Bush })}+c(i \omega)^{(\text {Bush })}} & 0 \\ 0 & \frac{1}{k^{(\text {Bush })}+c(i \omega)^{(\text {Bush })}}\end{array}\right]$.

As previous in Eq. 9, the dynamic equations for the uncoupled system (7) and the reformed compatibility condition (13) can be written in block matrix form

$\left[\begin{array}{cc}\boldsymbol{Z} & \boldsymbol{B}^{T} \\ \boldsymbol{B} & -\boldsymbol{\Gamma}\end{array}\right]\left[\begin{array}{l}\boldsymbol{u} \\ \boldsymbol{\lambda}\end{array}\right]=\left[\begin{array}{l}\boldsymbol{f} \\ \mathbf{0}\end{array}\right]$.

Note that, from a mathematical point of view, considering the interface flexibility of the bushings can be seen as a pertubation method for the constraints $\boldsymbol{B} \boldsymbol{u}=\mathbf{0}$. The dual interface problem can now be formed, as shown in Section "Frequency-Based Substructuring", to compute the interface Lagrange multipliers:

$\lambda=\left(\boldsymbol{B} \boldsymbol{Y} \boldsymbol{B}^{T}+\boldsymbol{\Gamma}\right)^{-1} \boldsymbol{B} \boldsymbol{Y} \boldsymbol{f}$
Inserting the Lagrange multiplier vector $\lambda$ into the admittance form of the dynamic (8), one obtains the system assembly admittance when considering the compatibility relaxation

$\boldsymbol{Y}_{\text {assembled }}=\boldsymbol{Y}-\boldsymbol{Y} \boldsymbol{B}^{T}\left(\boldsymbol{B} \boldsymbol{Y} \boldsymbol{B}^{T}+\boldsymbol{\Gamma}\right)^{-1} \boldsymbol{B} \boldsymbol{Y}$

This method can be combined very well with different damping models. Both frequency-dependent damping models such as viscous damping and frequency-independent models such as hysteretic damping can be used. In literature [7], it is mentioned that this method can also be adapted for nonlinear models such as the Jenkins model [9] or an Iwan model [10].

\section{Bushings as Substructure by Adding Virtual Masses}

In this method, rubber bushings can be integrated into the classical LM-FBS (11) through a regularization of the impedance matrix $\boldsymbol{Z}$ by adding virtual masses (see Fig. 3). This allows the typical characteristic of singularity of a dynamic impedance to be bypassed such that it is possible to treat the bushing as a regular substructure:

$\boldsymbol{Y}^{(\text {Bush) }}=\left(\boldsymbol{K}^{(\text {Bush) }}+\boldsymbol{C}(\boldsymbol{i} \boldsymbol{\omega})^{(\text {Bush) }}-\omega^{2} \boldsymbol{M}^{(\text {Bush })}\right)^{-1}$

Since the system is modified by adding additional mass, the following possible approaches should be considered [8]:

- The virtual mass matrix $M^{\text {(Bush) }}$ should have small entries. To make sure that the frequency range of interest of the assembly will not be affected too much, the mass perturbation, as a rule of thumb, should be selected so that the local non-zero eigenfrequencies of the bushing are at least 10 times above the frequency of interest of the components.

- The effect of the additional mass can be canceled out by coupling another virtual substructure with a negative mass. With this method the mass can be chosen arbitrarily.

- The effect of the lumped masses added to the bushing can also be canceled out by directly removing them in the mass matrix of the components (if the matrices of the components are known).

In the context of this work, the first of the three methods was chosen for simplicity's sake.
Fig. 2 Assembly of two substructures with bushings as compatibility relaxation in between

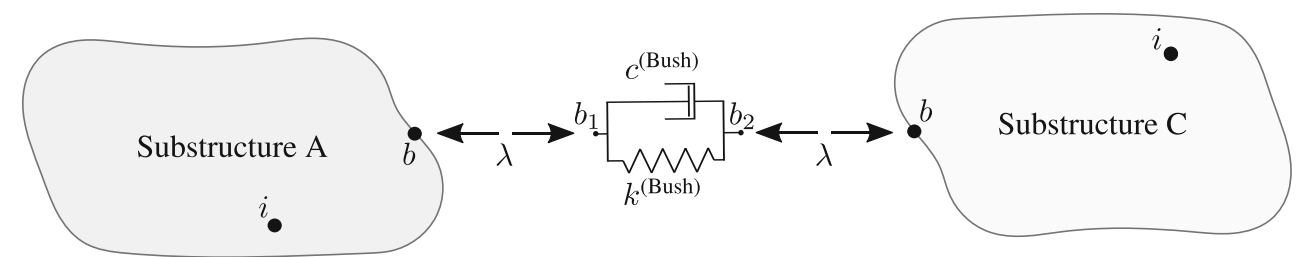


Fig. 3 Assembly of substructures with bushings implemented by adding virtual masses

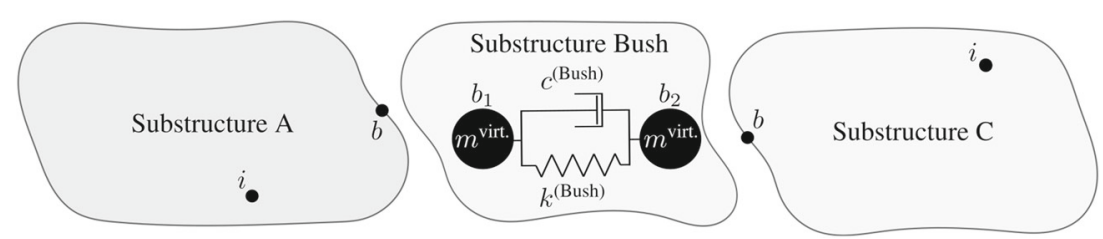

\section{Bushing as Substructure via Pseudoinverse}

A final method is presented which generalizes the classical LM-FBS formulation, so that actual singular matrices can now also be integrated into the process. Figure 4 shows a system consisting of substructures $\mathrm{A}$ and $\mathrm{C}$, with a rubber bearing arranged between them and also considered as a substructure. The dynamic stiffness $Z^{\text {Bush }}$ for the rubber bushing element from the illustrated model can thus be set up as

$Z^{(\text {Bush })}=\left[\begin{array}{ll}k^{(\text {Bush })}+c(i \omega)^{(\text {Bush })} & -k^{(\text {Bush })}-c(i \omega)^{(\text {Bush })} \\ -k^{(\text {Bush })}-c(i \omega)^{(\text {Bush })} & k^{(\text {Bush })}+c(i \omega)^{(\text {Bush })}\end{array}\right]$.

The fact that $\boldsymbol{Z}^{\text {Bush }}$ is singular means that the dynamics of the bushing substructure

$\boldsymbol{Z}^{(\text {Bush) }} \boldsymbol{u}^{\text {(Bush) }}=\boldsymbol{f}^{\text {(Bush) }}-\boldsymbol{B}^{\left(\text {Bush) }{ }^{T}\right.} \lambda$

offer a solution only if all applied forces are selfequilibrated or, in other words, if the applied forces have zero resulting forces and moments. Mathematically, this is expressed by the fact that the applied forces must not produce any work on the rigid body modes $\boldsymbol{R}[11,12]$ :

$\boldsymbol{R}^{(\text {Bush })^{T}}\left(f^{(\text {Bush })}-B^{\left(\text {(Bush }^{T}\right.} \lambda\right)=\mathbf{0}$.

In consideration of the condition given in Eq. 21, there will be a solution for the DOFs of the rubber bushings

$\boldsymbol{u}^{(\text {Bush) }}=\boldsymbol{Y}^{(\text {Bush) }}\left(f^{(\text {Bush })}-B^{(\text {Bush })^{T}} \lambda\right)-\boldsymbol{R}^{(\text {Bush) }} \boldsymbol{\alpha}^{(\text {Bush })}$,

where $\boldsymbol{\alpha}^{\text {(Bush) }}$ represents the amplitudes of the rigid body components of the solution, that are left undetermined by Eq. 22. In order to get an admittance $\boldsymbol{Y}^{\text {(Bush) }}$ of the substructure Bush depicted in Fig. 4, the pseudoinverse of the dynamic stiffness matrix has to be considered. It can be computed either by singular value decomposition (SVD) or, more efficiently, by an incomplete factorization as described in [11]. For instance, in the case the bushing connects only one degree of freedom on each side (Fig. 4 and Eq. 19), it can be computed in the form of

$\boldsymbol{Y}^{(\mathrm{Bush})}=\left(\boldsymbol{Z}^{(\mathrm{Bush})}\right)^{+}=\left[\begin{array}{cc}\frac{1}{k^{(\mathrm{Bush})}+c(i \omega)^{(\mathrm{Bush})}} & 0 \\ 0 & 0\end{array}\right]$.

The general solution of an assembly having several bushing substructures can be written as

$\boldsymbol{u}=\boldsymbol{Y}\left(\boldsymbol{f}-\boldsymbol{B}^{T} \lambda\right)-\boldsymbol{R} \boldsymbol{\alpha}$.

The rigid body modes $\boldsymbol{R}^{(\text {Bush) }}$ and their corresponding amplitudes $\boldsymbol{\alpha}^{\text {(Bush) }}$ are defined as

$\begin{aligned} \boldsymbol{R} & =\left[\begin{array}{cccc}\mathbf{0} & \cdots & \cdots & \mathbf{0} \\ \vdots & \ddots & & \vdots \\ \boldsymbol{R}^{(\text {Bush1) }} & \mathbf{0} & & \vdots \\ \mathbf{0} & \boldsymbol{R}^{(\text {Bush2) }} & & \vdots \\ \vdots & & \ddots & \mathbf{0} \\ \mathbf{0} & \cdots & \mathbf{0} & \boldsymbol{R}^{(\text {BushN })} \\ \vdots & \cdots & & \vdots \\ \mathbf{0} & \cdots & & \mathbf{0}\end{array}\right], \\ \boldsymbol{\alpha} & =\left[\begin{array}{c}\boldsymbol{\alpha}^{(\text {Bush1) }} \\ \boldsymbol{\alpha}^{\text {(Bush2) }} \\ \vdots \\ \boldsymbol{\alpha}^{(\text {BushN) }}\end{array}\right] .\end{aligned}$

For the one dimensional example in Fig. 4, the setup of the dynamic equation in the uncoupled system would look like

$u=Y(f+g)-R \alpha$
Fig. 4 Assembly of substructures with bushings implemented by pseudoinverse method

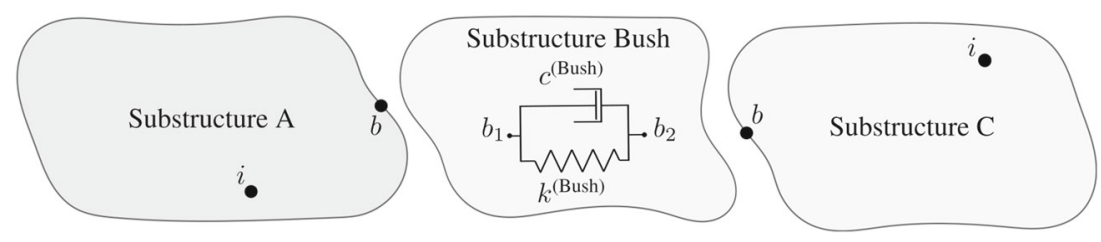




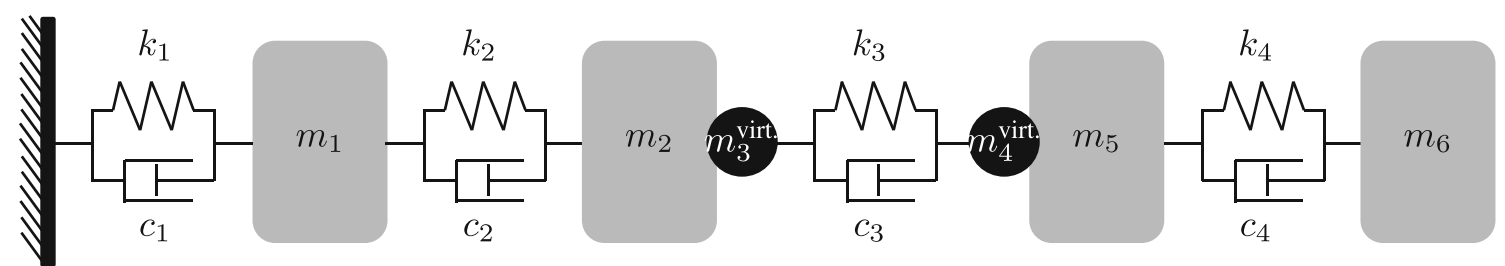

Fig. 5 Schematic representation of the minimal example

$$
\begin{aligned}
{\left[\begin{array}{c}
\boldsymbol{u}_{i}^{(\mathrm{A})} \\
\boldsymbol{u}_{b}^{(\mathrm{A})} \\
\boldsymbol{u}_{\mathrm{b} 1}^{(\mathrm{Bush})} \\
\boldsymbol{u}_{\mathrm{b} 2}^{(\mathrm{Bush})} \\
\boldsymbol{u}_{b}^{(\mathrm{C})} \\
\boldsymbol{u}_{i}^{(\mathrm{C})}
\end{array}\right]=} & {\left[\begin{array}{cccccc}
\boldsymbol{Y}_{i i}^{(\mathrm{A})} & \boldsymbol{Y}_{i b}^{(\mathrm{A})} & \mathbf{0} & \mathbf{0} & \mathbf{0} & \mathbf{0} \\
\boldsymbol{Y}_{b i}^{(\mathrm{A})} & \boldsymbol{Y}_{b b}^{(\mathrm{A})} & \mathbf{0} & \mathbf{0} & \mathbf{0} & \mathbf{0} \\
\mathbf{0} & \mathbf{0} & \boldsymbol{Y}_{\mathrm{b} 1}^{(\mathrm{Bush})} & \mathbf{0} & \mathbf{0} & \mathbf{0} \\
\mathbf{0} & \mathbf{0} & \mathbf{0} & \boldsymbol{Y}_{\mathrm{b} 2}^{(\mathrm{Bush})} & \mathbf{0} & \mathbf{0} \\
\mathbf{0} & \mathbf{0} & \mathbf{0} & \mathbf{0} & \boldsymbol{Y}_{b b}^{(\mathrm{C})} & \boldsymbol{Y}_{b i}^{(\mathrm{C})} \\
\mathbf{0} & \mathbf{0} & \mathbf{0} & \mathbf{0} & \boldsymbol{Y}_{i b}^{(\mathrm{C})} & \boldsymbol{Y}_{i i}^{(\mathrm{C})}
\end{array}\right] } \\
& \left.\times\left[\begin{array}{c}
\boldsymbol{f}_{i}^{(\mathrm{A})} \\
\boldsymbol{f}_{b}^{(\mathrm{A})} \\
\boldsymbol{f}_{\mathrm{b} 1}^{(\mathrm{Bush})} \\
\boldsymbol{f}_{\mathrm{b} 2}^{(\mathrm{Bush})} \\
\boldsymbol{f}_{b}^{(\mathrm{C})} \\
\boldsymbol{f}_{i}^{(\mathrm{C})}
\end{array}\right]+\left[\begin{array}{c}
\mathbf{0} \\
\boldsymbol{g}_{b}^{(\mathrm{A})} \\
\boldsymbol{g}_{\mathrm{b} 1}^{(\mathrm{Bush})} \\
\boldsymbol{g}_{\mathrm{b} 2}^{(\mathrm{Bush})} \\
\boldsymbol{g}_{b}^{(\mathrm{C})} \\
\mathbf{0}
\end{array}\right]\right]-\left[\begin{array}{l}
\mathbf{0} \\
\mathbf{0} \\
1 \\
1 \\
\mathbf{0} \\
\mathbf{0}
\end{array}\right] \boldsymbol{\alpha}^{(\mathrm{Bush})} .
\end{aligned}
$$

Taking into account the general uncoupled solution (26), the compatibility condition (5) and the self-equilibrium condition (21), the system of equations can be summarized as follows

$$
\left[\begin{array}{ccc}
\boldsymbol{I} & \boldsymbol{Y} \boldsymbol{B}^{T} & \boldsymbol{R} \\
\boldsymbol{B} & \mathbf{0} & \mathbf{0} \\
\mathbf{0} & \boldsymbol{R}^{T} \boldsymbol{B}^{T} & \mathbf{0}
\end{array}\right]\left[\begin{array}{c}
\boldsymbol{u} \\
\boldsymbol{\lambda} \\
\boldsymbol{\alpha}
\end{array}\right]=\left[\begin{array}{c}
\boldsymbol{Y} \boldsymbol{f} \\
\mathbf{0} \\
\boldsymbol{R}^{T} \boldsymbol{f}
\end{array}\right] .
$$

Eliminating the degrees of freedom of the substructures, the dual interface problem to compute the interface Lagrange multipliers $\lambda$ and $\boldsymbol{\alpha}$ can now be obtained as

$$
\left[\begin{array}{l}
\lambda \\
\boldsymbol{\alpha}
\end{array}\right]=\left[\begin{array}{ccc}
\boldsymbol{B} \boldsymbol{Y} \boldsymbol{B}^{T} & \boldsymbol{B} \boldsymbol{R} \\
\boldsymbol{R}^{T} & \boldsymbol{B}^{T} & \mathbf{0}
\end{array}\right]^{-1}\left[\begin{array}{l}
\boldsymbol{B} \boldsymbol{Y} \\
\boldsymbol{R}^{T}
\end{array}\right] f .
$$

Substituting the solution into the governing equation then returns the dually assembled admittance

$$
\boldsymbol{Y}_{\text {assembled }}=\boldsymbol{Y}-\left[\begin{array}{lll}
\boldsymbol{Y} & \boldsymbol{B}^{T} & \boldsymbol{R}
\end{array}\right]\left[\begin{array}{ccc}
\boldsymbol{B} \boldsymbol{Y} \boldsymbol{B}^{T} & \boldsymbol{B} \boldsymbol{R} \\
\boldsymbol{R}^{T} \boldsymbol{B}^{T} & \mathbf{0}
\end{array}\right]^{-1}\left[\begin{array}{c}
\boldsymbol{B} \boldsymbol{Y} \\
\boldsymbol{R}^{T}
\end{array}\right]
$$

so that the assembled response can be computed by

$\boldsymbol{u}_{\text {assembled }}=\boldsymbol{Y}_{\text {assembled }} \boldsymbol{f}$.

This Eq. 29 represents an extended generalized formulation of the LM-FBS approach, which considers also singular substructures, as it is the case for rubber bushings. For the explicit coupling of non singular substructures, this formulation can be used as well, since the matrix $\boldsymbol{R}$ would have only zero entries and it would result in the well-known classical LM-FBS (11).

\section{Comparison of All Three Methods on a Minimal Example}

Now that three different methods have been presented to introduce rubber bushings into the LM-FBS process, a minimal example (Fig. 5) will be used to show that all methods behave approximately the same. Figure 6 shows the analytically calculated transfer function of the assembled system and their deviations in relation to the transfer function for the compatibility relaxation method. Thereby the parameters listed in Table 1 were used. The
Fig. 6 Comparison of three coupling methods:

Compatibility relaxation, Virtual masses and Pseudoinverse. In addition, the coherence between the different methods is presented

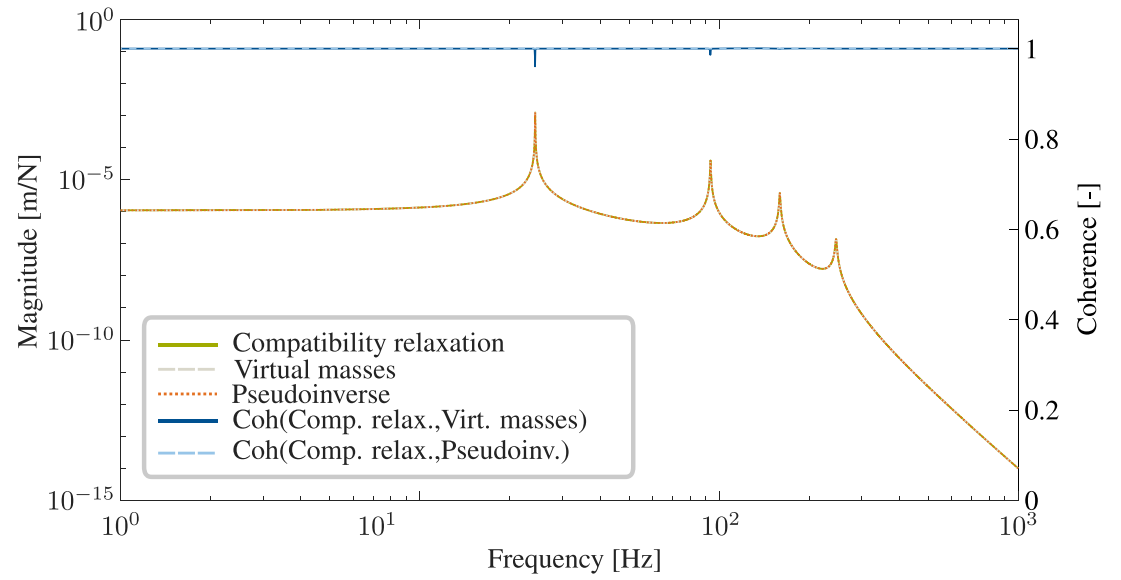


Table 1 Parameters for the minimal example of the three coupling methods. Rayleigh damping was assumed with mass and stiffness proportional factor of $\alpha=0$ and $\beta=3.8 \times 10^{-5}$

\begin{tabular}{|c|c|c|c|c|c|c|c|c|c|}
\hline \multirow[t]{2}{*}{ Substructure } & \multicolumn{3}{|c|}{ Compatibility relaxation } & \multicolumn{3}{|c|}{ Virtual masses } & \multicolumn{3}{|c|}{ Pseudo-inverse } \\
\hline & A & Bush & $\mathrm{C}$ & A & Bush & $\mathrm{C}$ & A & Bush & $\mathrm{C}$ \\
\hline \multirow[t]{2}{*}{ mass } & $m_{1}$ & massless & $m_{5}$ & $m_{1}$ & $m_{3}^{\text {virt. }}$ & $m_{5}$ & $m_{1}$ & massless & $m_{5}$ \\
\hline & $m_{2}$ & & $m_{6}$ & $m_{2}$ & $m_{4}^{\text {virt. }}$ & $m_{6}$ & $m_{2}$ & & $m_{6}$ \\
\hline \multirow[t]{2}{*}{ stiffness } & $k_{1}$ & $k_{3}$ & $k_{4}$ & $k_{1}$ & $k_{3}$ & $k_{4}$ & $k_{1}$ & $k_{3}$ & $k_{4}$ \\
\hline & $k_{2}$ & & & $k_{2}$ & & & $k_{2}$ & & \\
\hline \multirow[t]{4}{*}{ parameter } & mass in & \multicolumn{8}{|c|}{$m_{1}=30, m_{2}=50, m_{3}^{\text {virt. }}=0.05$} \\
\hline & {$[k g]$} & \multicolumn{8}{|c|}{$m_{4}^{\text {virt. }}=0.05, m_{5}=70, m_{6}=60$} \\
\hline & stiffness in & \multicolumn{8}{|c|}{$k_{1}=9, k_{2}=7$} \\
\hline & {$\left[10^{5} \mathrm{~N} / \mathrm{m}\right]$} & \multicolumn{5}{|c|}{$k_{3}=1, k_{4}=8$} & & & \\
\hline
\end{tabular}
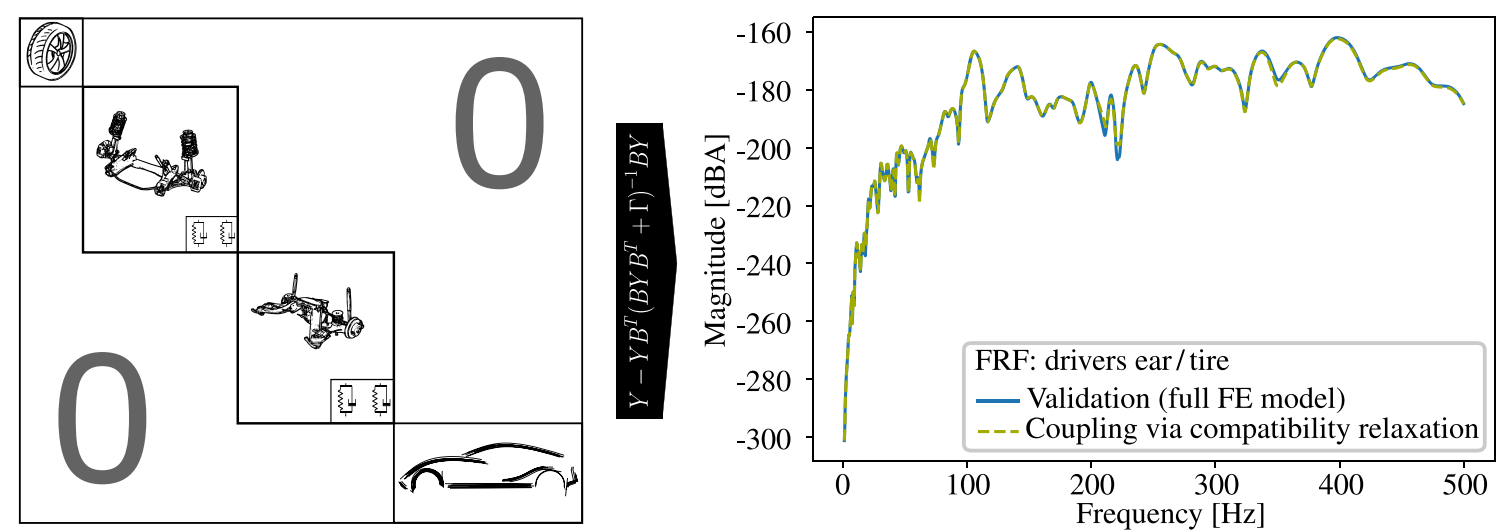

Fig. 7 Introduction of bushings as compatibility relaxation to the total vehicle system
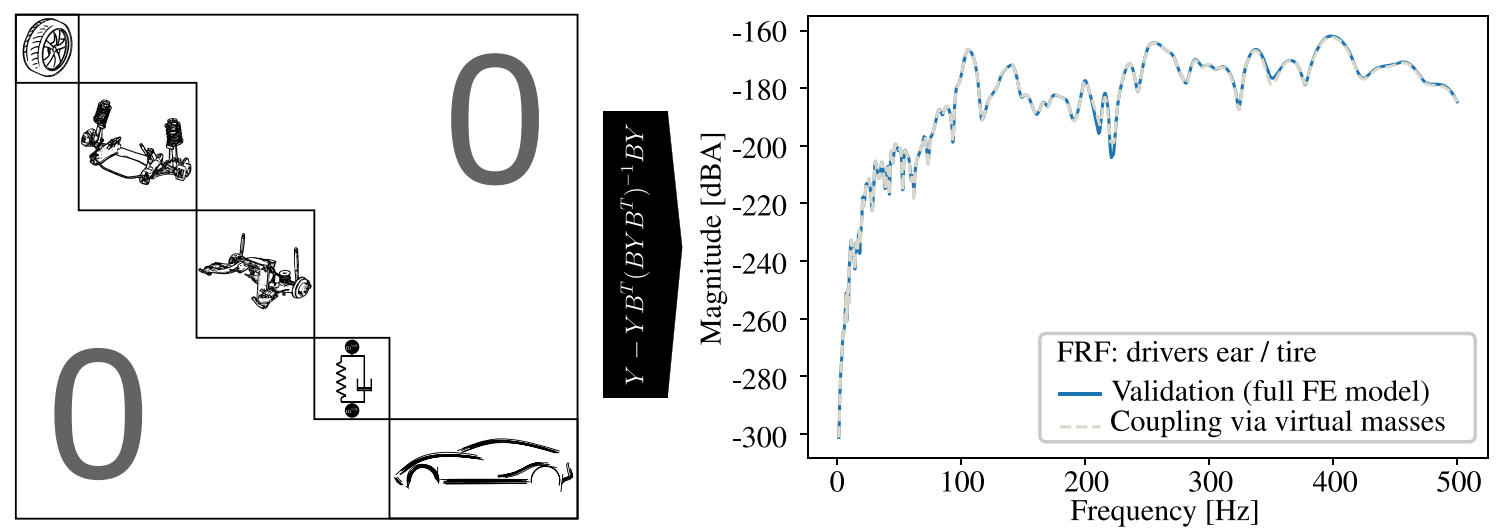

Fig. 8 Introduction of bushings as substructure to the total vehicle system by adding virtual masses 
force was applied at the internal DOF $\left(m_{1}\right)$ of substructure $\mathrm{A}$ and the response was considered at the internal DOF $\left(m_{6}\right)$ of substructure $\mathrm{C}$.

\section{Application of the Methods on Simulated Vehicle Subsystems}

This section provides an insight into the practical application of the methods presented in Section "Introduction of Bushings in the General Framework of LM-FBS". Simulated FRF data of a total vehicle system and of its components were provided. These could now be used to couple the individual substructures of the car using the LM-FBS method. The substructures to be coupled were four tires, a front suspension, a rear suspension, the rubber bushings and the body in white (BIW). The simulation of the structure borne sound of the BIW was performed with a vibroacoustic FE model that is available as a standard model in commercial codes. The air in the interior was simulated by a linear acoustic model (Helmoltz equations) and coupled to the vibration of the structure. The main focus here was on the rubber mounts, which were attached to the corresponding DOFs between front suspension and BIW, and rear suspension and BIW. Considering hysteretic damping at the interfaces, i.e. $c(i \omega)=i c^{\text {Bush }}$ where $c^{\text {Bush }}$ is constant, the bushings were implemented in all presented methods.

Figure 7 shows an illustration of the uncoupled admittance matrix $\boldsymbol{Y}$ on the left. Here the individual admittances of the substructures are arranged in block diagonal form. The rubber bushings in the lower right corner of the front and rear suspension block should represent the compatibility relaxation at the interfaces. By applying the corresponding adapted LM-FBS formula, taking into account the interface relaxation, the plot on the right side results. The plot shows the frequency response function (FRF) of the assembled total vehicle system when no kinematic boundary conditions are applied on the assembled system. The FRF shown in Fig. 7 corresponds to an excitation at the point of contact to the road of the tire which results in a sound pressure level response at the drivers ear in the interior of the car. The validation is the same in all plots and was created in a commercial case as a complete assembled model. It can be clearly seen that the coupling via compatibility relaxation matches perfectly, as expected from the theory, with the validation response, except of few small deviations at certain frequencies that are not due to the coupling method, but rather to the specific manner bushings are considered in the commercial code.

In Fig. 8 bushings are introduced as substructure by adding virtual lumped masses. These masses were chosen that the first non-zero eigenfrequencies of the bushings are at least 10 times above the frequency of interest of the components. In Fig. 9 bushings are also introduced as substructures but now via the pseudoinverse method. Only very small differences between the full simulation and the FBS solution can be observed for the method of virtual masses.

\section{Conclusions}

In this paper, a derivation and comparison of three different methods was shown to handle singular substructures, as it is the case of rubber bushings, in the LM-FBS approach. The investigated methods are the introduction of the bushing as compatibility relaxation, treating them as a substructure by adding virtual masses and also as substructure, but then via a pseudoinverse approach. The most elegant method offers the introduction as substructure with the use of the pseudoinverse. This method represents an extended generalized formulation of the LM-FBS approach, which considers also singular substructures, as is the case for rubber bushings. At the same time, this method is also
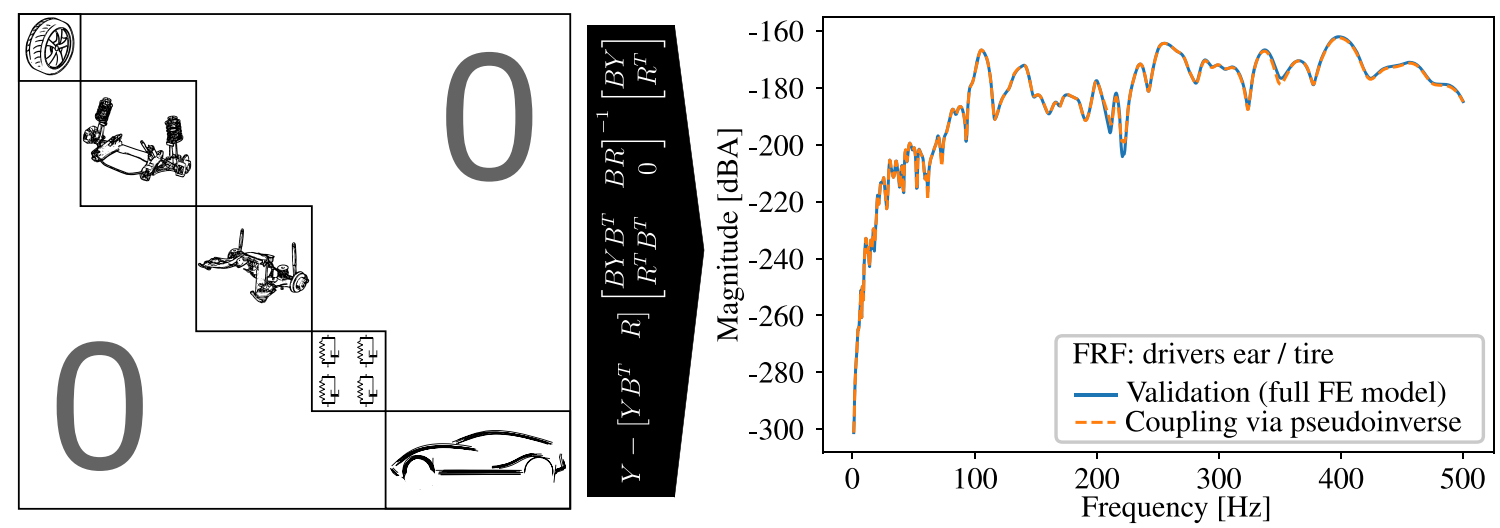

Fig. 9 Introduction of bushings as substructure to the total vehicle system via pseudoinverse method 
suitable for non-singular matrices. Finally, the methods could also be applied to the practical example of a total vehicle system.

Acknowledgments Open Access funding provided by Projekt DEAL. We want to thank Dr. Yong-Dae Kim from the Hyundai Motor Company for providing us the simulated data of the total vehicle system.

\section{Compliance with Ethical Standards}

Conflict of interests On behalf of all authors, the corresponding author states that there is no conflict of interest.

Open Access This article is licensed under a Creative Commons Attribution 4.0 International License, which permits use, sharing, adaptation, distribution and reproduction in any medium or format, as long as you give appropriate credit to the original author(s) and the source, provide a link to the Creative Commons licence, and indicate if changes were made. The images or other third party material in this article are included in the article's Creative Commons licence, unless indicated otherwise in a credit line to the material. If material is not included in the article's Creative Commons licence and your intended use is not permitted by statutory regulation or exceeds the permitted use, you will need to obtain permission directly from the copyright holder. To view a copy of this licence, visit http:// creativecommonshorg/licenses/by/4.0/.

\section{References}

1. Craig RR, Bampton MCC (1968) Coupling of substructures for dynamic analyses. In: AIAA journal 6.7, pp 1313-1319. https://doi.org/10.2514/3.4741

2. Gordis JH, Bielawa RL, Flannelly WGA (1991) General theory for frequency domain structural synthesis. In: Journal of sound and vibration 150.1, pp 139-158
3. de Klerk D, Rixen DJ, Voormeeren SN (2008) General framework for dynamic substructuring: history, review and classification of techniques. In: AIAA journal 46.5, pp 1169-1181. https://doi.org/10.2514/1.33274

4. Zeller P (2012) Handbuch Fahrzeugtechnik: Grundlagen, Auslegung, Berechnung, Versuch. ATZ-MTZ Fachbuch. Vieweg + Teubner

5. Jetmundsen B, Bielawa RL, Flannelly WG (1988) Generalized frequency domain substructure synthesis. In: Journal of the american helicopter society 33.1 , pp 55-64

6. de Klerk D, Rixen DJ, de Jong J (2006) The frequency based substructuring (FBS) method reformulated according to the dual domain decomposition method. In: 24th international modal analysis conference, St. Louis, MO

7. Barten E, van der Seijs M, de Klerk D (2014) A complex power approach to characterise joints in experimental dynamic substructuring. In: Dynamics of coupled structures, vol 6: Proceedings of the 32nd IMAC, a conference and exposition on structural dynamics. Springer, New York, pp 281-296

8. Allen M, Rixen DJ, Tiso P, Mayes R, van der Seijs M, Abrahamson T (2018) Substructuring in engineering dynamics emerging numerical and experimental techniques. Lecture notes of CISM, pp 62-78

9. Bograd S, Reuss P, Schmidt A, Gaul L, Mayer M (2011) Modeling the dynamics of mechanical joints. In: Mechanical systems and signal processing 25.8, pp 2801-2826. https://doi.org/10.1016/j. ymssp.2011.01.010

10. Iwan WD (1966) A distributed-element model for hysteresis and its steady-state dynamic response. In: Journal of applied mechanics 33.4, p 893. https://doi.org/10.1115/1.3625199

11. Geradin M, Rixen DJ (2015) Mechanical vibrations: theory and application to structural dynamics. Wiley, New York

12. Farhat C, Roux F (1991) Method o f finite element tearing and interconnecting and its parallel solution algorithm. In: International journal for numerical methods in engineering 32, pp 1205-1227

Publisher's Note Springer Nature remains neutral with regard to jurisdictional claims in published maps and institutional affiliations. 